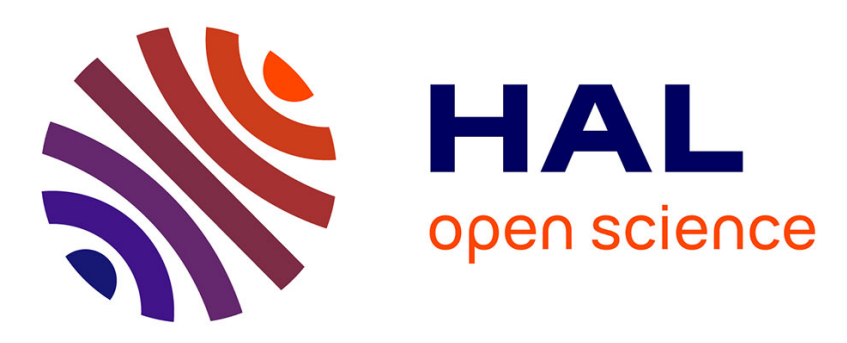

\title{
Second-Order Horizontal Synchrosqueezing of the S-transform: a Specific Wavelet Case Study
}

Dominique Fourer, François Auger

\section{To cite this version:}

Dominique Fourer, François Auger. Second-Order Horizontal Synchrosqueezing of the S-transform: a Specific Wavelet Case Study. 28th European Signal Processing Conference (EUSIPCO 2020), Jan 2021, Amsterdam, Netherlands. pp.2200-2204, 10.23919/Eusipco47968.2020.9287573 . hal-02889193

\section{HAL Id: hal-02889193 https://hal.science/hal-02889193}

Submitted on 3 Jul 2020

HAL is a multi-disciplinary open access archive for the deposit and dissemination of scientific research documents, whether they are published or not. The documents may come from teaching and research institutions in France or abroad, or from public or private research centers.
L'archive ouverte pluridisciplinaire HAL, est destinée au dépôt et à la diffusion de documents scientifiques de niveau recherche, publiés ou non, émanant des établissements d'enseignement et de recherche français ou étrangers, des laboratoires publics ou privés. 


\section{Second-Order Horizontal Synchrosqueezing of the S-transform: a Specific Wavelet Case Study}

\author{
Dominique Fourer \\ IBISC Univ. Évry/Paris-Saclay \\ Courcouronnes, France \\ dominique.fourer@univ-evry.fr
}

\author{
François Auger \\ IREENA, Univ. Nantes \\ Saint-Nazaire, France \\ francois.auger@univ-nantes.fr
}

\begin{abstract}
We address the problem of computing efficient time-frequency and time-scale representations of non stationary multicomponent signals. Recently, a new time-reassigned synchrosqueezing method designed for the short-time Fourier transform (STFT) was introduced to improve the energy concentration of impulsive or strongly modulated signals. Following this idea, we now propose to extend this approach to the Stockwell transform (S-transform) that is related to the Morlet wavelet transform and which provides time-frequency representations with a frequency-dependent resolution. In this study, we derive an enhanced second-order group delay estimator designed for the S-transform to deal with strongly amplitude- and frequencymodulated signals. Hence, this estimator is used to obtain a novel horizontal synchrosqueezing transform that is evaluated in numerical experiments involving multicomponent non-stationary signals.

Index Terms -S-transform, wavelet, time-frequency, horizontal synchrosqueezing, group-delay estimation
\end{abstract}

\section{INTRODUCTION}

Natural signals arising from audio, biomedicine, seismic or radar can be modeled as a mixture of non-stationary components that must be disentangled through a suitable methodology. Among popular methods, time-frequency (resp. time-scale) analysis methods with the Short-Time Fourier Transform (STFT) and the Continuous Wavelet Transform (CWT) offer an efficient framework for efficiently representing a large variety of signals [1]. Due to several limitations which can mostly be explained by the Heisenberg-Gabor uncertainty principle, several promising post-processing methods were introduced to enhance the readability of a time-frequency representation (TFR). The reassignment [2], [3] and the synchrosqueezing methods [4], [5] were proposed to sharpen a TFR by moving the transforms values to more accurate coordinates which are close to the exact time-frequency support of the signal, resulting in an enhanced readability. The current renew of interest for such techniques can be explained by the capability of the synchrosqueezing to compute reversible TFRs which paves the way of advanced processing methods for disentangling and extracting the elementary signal components (modes) [6], [7]. Nowadays, synchrosqueezing is continuously improved by several extensions and theoretical studies allowing to deal with amplitude- and frequency-modulated

This research was supported by the ORACLES Paris-Saclay research project and the French ANR ASCETE project. signals [8], [9], [10], [11]. In [12], [13], the LevenbergMarquardt algorithm is used to make the reassignment and the synchrosqueezing adaptive and more robust to noise through a damping parameter. These methods have shown their potential for disentangling multicomponent non-stationary signals and to extract physically interpretable atoms [6].

In this study, we introduce an enhanced second-order group delay estimator designed for the Stockwell transform (also called S-transform) [14] allowing the computation of a recently introduced method called time-reassigned synchrosqueezing [15]. The S-transform can be considered as a special case of the Morlet wavelet transform and can be viewed as a frequency-dependent STFT, as the constant-Q transform [16]. It is a full of interest time-frequency transform for theoretical investigations and practical applications. Hence, the present work introduces for the S-transform a new specific secondorder horizontal synchrosqueezing technique and extends our previously proposed method presented in [8].

The paper is organized as follows. The S-transform is presented in Section II with its properties and its relations with the CWT. In Section III, we recall the principle of the reassignment method and we introduce the time-reassigned synchrosqueezed S-transform before the derivation of a group delay estimator designed for computing a novel second-order horizontal synchrosqueezed S-transform. In Section V, we present numerical results of the proposed methods for efficiently computing TFRs before concluding the paper with future work directions in Section VI.

\section{S-TRANSFORM DEFINITION AND PROPERTIES}

\section{A. Definition}

The S-transform of a zero-mean signal $x$ can be defined at any time $t$ and frequency $\omega$ as a STFT using a frequencydependent analysis window $h$, expressed as [14]:

$$
\operatorname{ST}_{x}^{h}(t, \omega)=\frac{|\omega|}{\omega_{0}} \int_{-\infty}^{+\infty} x(\tau) h\left(\frac{\omega}{\omega_{0}}(t-\tau)\right)^{*} \mathbf{e}^{-j \omega \tau} d \tau
$$

where $j^{2}=-1$ and $z^{*}$ is the complex conjugate of $z$. In the original version (considered in this work), $h$ is chosen as a Gaussian window parameterized by a time-spread parameter $T$ as $h(t)=g(t, T)=\frac{1}{\sqrt{2 \pi} T} \mathbf{e}^{-\frac{t^{2}}{2 T^{2}}}$, enabling us to write: 


$$
\begin{aligned}
\operatorname{ST}_{x}^{g}(t, \omega) & =\frac{|\omega|}{\omega_{0}} \int_{-\infty}^{+\infty} x(\tau) g\left(\frac{|\omega|}{\omega_{0}}(t-\tau), T\right)^{*} \mathbf{e}^{-j \omega \tau} d \tau \\
& =\mathbf{e}^{-j \omega t} \int_{-\infty}^{+\infty} x(t+\tau) g\left(-\tau, \frac{\omega_{0} T}{|\omega|}\right)^{*} \mathbf{e}^{-j \omega \tau} d \tau
\end{aligned}
$$

where $T$ corresponds to the width of the Gaussian window $g$ when $\omega=\omega_{0}$. This allows us to derive mathematical expressions involving only an adimensional parameter $f_{0} T=\frac{1}{2 \pi} \omega_{0} T$ where $f_{0}$ is a central frequency parameter expressed in $\mathrm{Hz}$. The S-transform can also be computed in the frequency domain using the following expression that can be deduced from the Plancherel theorem (detailed proof in [17]):

$$
\mathrm{ST}_{x}^{g}(t, \omega)=\int_{-\infty}^{+\infty} F_{x}(\omega+\Omega) F_{h}\left(\frac{\omega_{0}}{\omega} \Omega\right)^{*} \mathbf{e}^{j \Omega t} \frac{d \Omega}{2 \pi}
$$

where $F_{x}$ denotes the Fourier transform of signal $x$ and:

$$
F_{h}(\omega)=\int_{-\infty}^{+\infty} h(t) \mathbf{e}^{-j \omega t} d t=\mathbf{e}^{-\frac{\omega^{2} T^{2}}{2}} .
$$

As for the spectrogram and the scalogram, computed by squaring the modulus of the transform, a TFR called stockwellogram is defined as $\left|\operatorname{ST}_{x}(t, \omega)\right|^{2}$.

\section{B. Marginalization and inversion formula}

Marginalization of the S-transform with respect to time leads to:

$$
\begin{aligned}
\int_{-\infty}^{+\infty} \mathrm{ST}_{x}^{g}(t, \omega) d t & =\iint_{\mathbb{R}^{2}} F_{x}(\omega+\Omega) F_{h}\left(\frac{\omega_{0}}{\omega} \Omega\right)^{*} \mathbf{e}^{j \Omega t} \frac{d \Omega}{2 \pi} d t \\
& =\int_{\mathbb{R}} F_{x}(\omega+\Omega) F_{h}\left(\frac{\omega_{0}}{\omega} \Omega\right)^{*} \delta(\Omega) d \Omega \\
& =F_{x}(\omega) F_{h}(0)^{*}
\end{aligned}
$$

that is equal to $F_{x}(\omega)$ when a Gaussian window is used (i.e. $F_{h}(0)=1$ ). Hence $x$ can finally be reconstructed by applying the inverse Fourier transform which leads us to:

$$
x(t)=\frac{1}{2 \pi} \iint_{\mathbb{R}^{2}} \operatorname{ST}_{x}^{g}(\tau, \omega) \mathbf{e}^{j \omega t} d \tau d \omega .
$$

\section{Relation with the continuous wavelet transform}

The CWT of a signal $x$ is defined at each time $t$ and scale $s$ for an admissible mother wavelet $\Psi$ (i.e. satisfying $C_{\Psi}=$ $\left.\int_{\mathbb{R}}\left|F_{\Psi}(\omega)\right|^{2} \frac{d \omega}{|\omega|}<+\infty\right)$ as [18]:

$$
W_{x}(t, s)=\frac{1}{\sqrt{|s|}} \int_{-\infty}^{+\infty} x(\tau) \Psi\left(\frac{\tau-t}{s}\right)^{*} d \tau
$$

If we define the scale as $s=\frac{\omega_{0}}{\omega}$, Eq. (10) can be expressed as a time-frequency transform as:

$$
\mathrm{CW}_{x}(t, \omega)=\sqrt{\frac{|\omega|}{\omega_{0}}} \int_{-\infty}^{+\infty} x(\tau) \Psi\left(\frac{\omega}{\omega_{0}}(\tau-t)\right)^{*} d \tau .
$$

Now, if we use the Morlet wavelet [18], [1] given by:

$$
\Psi(t)=\frac{\pi^{-1 / 4}}{\sqrt{T}} \mathbf{e}^{\frac{-t^{2}}{2 T^{2}}} \mathbf{e}^{j \omega_{0} t}
$$

we finally obtain $\operatorname{MW}_{x}(t, \omega)=$

$$
\begin{aligned}
& \sqrt{\frac{|\omega|}{\omega_{0} T \sqrt{\pi}}} \int_{-\infty}^{+\infty} x(\tau) \mathbf{e}^{-\frac{\omega^{2}(t-\tau)^{2}}{2\left(\omega_{0} T\right)^{2}}} \mathbf{e}^{-j \omega(\tau-t)} d \tau \\
= & \sqrt{\frac{2 \sqrt{\pi} \omega_{0} T}{|\omega|}} \mathbf{e}^{j \omega t} \operatorname{ST}_{x}^{g}(t, \omega)
\end{aligned}
$$

Thus, the S-transform can be expressed as:

$$
\mathrm{ST}_{x}^{g}(t, \omega)=\sqrt{\frac{|\omega|}{2 \sqrt{\pi} \omega_{0} T}} \mathbf{e}^{-j \omega t} \operatorname{MW}_{x}(t, \omega)
$$

which corresponds to the Morlet Wavelet transform multiplied by a frequency-dependent scaling factor which increases with the frequency. This is the reason why the S-transform can be considered as a special case of the wavelet transform [19].

\section{REASSIGNMENT AND SYNCHROSQUEEZING OF THE S-TRANSFORM}

\section{A. Reassignment of the Stockwellogram}

The reassignment method [2], [3] aims at sharpening a TFR by moving its energy from $(t, \omega)$ to $\left(\hat{t}_{x}, \hat{\omega}_{x}\right)$, to improve the readability of the analyzed signal. For the S-transform, the reassignment operators can be computed as follows ( $c f$. [17] for the detailed non-trivial proof):

$$
\begin{array}{cl}
\tilde{t}_{x}(t, \omega)=t-\frac{\mathrm{ST}_{x}^{\mathcal{T} g}(t, \omega)}{\mathrm{ST}_{x}^{g}(t, \omega)}, & \tilde{\omega}_{x}(t, \omega)=j \omega+\frac{\mathrm{ST}_{x}^{\mathcal{D} g}(t, \omega)}{\mathrm{ST}_{x}^{g}(t, \omega)}, \\
\hat{t}_{x}(t, \omega)=\operatorname{Re}\left(\tilde{t}_{x}(t, \omega)\right), & \hat{\omega}_{x}(t, \omega)=\operatorname{Im}\left(\tilde{\omega}_{x}(t, \omega)\right)
\end{array}
$$

with $\mathcal{T} g(t)=t g(t)$ and $\mathcal{D} g(t)=\frac{d g}{d t}(t)$.

Hence, the reassigned stockwellogram can finally be computed as $\mathbf{R}_{x}(t, \omega)=$

$$
\iint_{\mathbb{R}^{2}}\left|\mathbf{S T}_{x}^{g}(\tau, \Omega)\right|^{2} \delta\left(t-\hat{t}_{x}(\tau, \Omega)\right) \delta\left(\omega-\hat{\omega}_{x}(\tau, \Omega)\right) d \tau d \Omega
$$

\section{B. Time-reassigned Synchrosqueezed S-transform}

To tackle the non-reversibility of the reassignment method, synchrosqueezing proposes to move the signal transform instead of its squared modulus, to preserve the phase information of the original transform [5]. As firstly proposed for the STFT, we introduce here a new time-reassigned synchrosqueezed $\mathrm{S}$-transform that is deduced from the marginalization with respect to time ( $c f$. Section II-B) and which can be defined as:

$$
S_{x}^{g}(t, \omega)=\int_{\mathbb{R}} \operatorname{ST}_{x}^{g}(\tau, \omega) \delta\left(t-\hat{t}_{x}(\tau, \omega)\right) d \tau .
$$

where $\hat{t}_{x}(t, \omega)$ is a group delay estimator computed using Eq. (17). Hence, its marginalization with respect to time leads to:

$$
\begin{aligned}
\int_{\mathbb{R}} S_{x}^{g}(t, \omega) d t & =\iint_{\mathbb{R}^{2}} \operatorname{ST}_{x}^{g}(\tau, \omega) \delta\left(t-\hat{t}_{x}(\tau, \omega)\right) d \tau d t \\
& =\int_{\mathbb{R}} \operatorname{ST}_{x}^{g}(\tau, \omega) d \tau=F_{x}(\omega)
\end{aligned}
$$


which enables us to derive the following exact signal reconstruction formula:

$$
\hat{x}(t)=\iint_{\mathbb{R}^{2}} S_{x}^{g}(\tau, \omega) \mathbf{e}^{j \omega t} d \tau \frac{d \omega}{2 \pi} .
$$

As a result, the time-reassigned synchrosqueezed S-transform provides a sharpened and reversible TFR designed for dealing with impulsive or strongly modulated signals. It also allows to recover the signal components by applying a filtering operation as proposed for the STFT in [8].

\section{A SECOND-ORDER HORIZONTAL SYNCHROSQUEEZED S-TRANSFORM}

\section{A. Signal model and properties}

Now, we consider the AM/FM signal model expressed as:

$$
\begin{aligned}
x(t) & =\mathbf{e}^{\lambda_{x}(t)+j \phi_{x}(t)} \\
\text { with } \quad \lambda_{x}(t) & =l_{x}+\mu_{x} t+\nu_{x} \frac{t^{2}}{2} \\
\text { and } \quad \phi_{x}(t) & =\varphi_{x}+\omega_{x} t+\alpha_{x} \frac{t^{2}}{2}
\end{aligned}
$$

where $\lambda_{x}(t)$ and $\phi_{x}(t)$ are respectively the log-amplitude and the phase with $q_{x}=\nu_{x}+j \alpha_{x}$ and $p_{x}=\mu_{x}+j \omega_{x}$.

This signal model verifies $\frac{d x}{d t}(t)=\left(q_{x} t+p_{x}\right) x(t)$ which enables us to write $\frac{\partial \mathrm{ST}_{x}^{g}}{\partial t}(t, \omega)=\mathrm{ST}_{x}^{\mathcal{D} g}(t, \omega)=$

$$
\begin{aligned}
& -j \omega \mathbf{S T}_{x}^{g}(t, \omega)+\mathbf{e}^{-j \omega t} \int_{\mathbb{R}} \frac{d x}{d t}(t+\tau) g\left(-\tau, \frac{\omega_{0} T}{|\omega|}\right)^{*} \mathbf{e}^{-j \omega \tau} d \tau \\
= & -j \omega \mathbf{S T}_{x}^{g}(t, \omega)+\left(p_{x}+q_{x} t\right) \mathbf{S T}_{x}^{g}(t, \omega)-q_{x} \mathbf{S T}_{x}^{\mathcal{T} g}(t, \omega)
\end{aligned}
$$

When $\left|\mathbf{S T}_{x}^{g}(t, \omega)\right|>0$, dividing by $\mathrm{ST}_{x}^{g}(t, \omega)$ leads to:

$$
\begin{aligned}
\frac{\mathrm{ST}_{x}^{\mathcal{D} g}(t, \omega)}{\mathrm{ST}_{x}^{g}(t, \omega)} & =p_{x}-j \omega+q_{x}\left(t-\frac{\mathrm{ST}_{x}^{\mathcal{T} g}(t, \omega)}{\mathbf{S T}_{x}^{g}(t, \omega)}\right) \\
& =p_{x}-j \omega+q_{x} \tilde{t}_{x}(t, \omega)
\end{aligned}
$$

and finally we have $\tilde{\omega}_{x}(t, \omega)=p_{x}+q_{x} \tilde{t}_{x}(t, \omega)$. Differentiating again with respect to $t$ allows to obtain an estimator $\hat{q}_{x}(t, \omega)=\frac{\partial \tilde{\omega}_{x}(t, \omega)}{\partial t} / \frac{\partial \tilde{t}_{x}(t, \omega)}{\partial t}$ that can be computed using several S-transforms with specific windows:

$$
\hat{q}_{x}(t, \omega)=\frac{\left(\mathbf{S T}_{x}^{\mathcal{D} g}(t, \omega)\right)^{2}-\mathbf{S T}_{x}^{\mathcal{D}^{2} g}(t, \omega) \mathbf{S T}_{x}^{g}(t, \omega)}{\mathbf{S T}_{x}^{\mathcal{T} \mathcal{D} g}(t, \omega) \mathbf{S T}_{x}^{g}(t, \omega)-\mathbf{S T}_{x}^{\mathcal{T} g}(t, \omega) \mathbf{S T}_{x}^{\mathcal{D} g}(t, \omega)}
$$

where $\mathcal{D}^{2} g(t, T)=\frac{d^{2} g}{d t}(t, T)$ and $\mathcal{T} \mathcal{D} g(t, T)=t \frac{d g}{d t}(t, T)$.

\section{$B$. Enhanced second-order group delay estimator}

Second-order horizontal synchrosqueezing [8] consists in moving $\mathrm{ST}_{x}^{g}(t, \omega)$ from the point $(t, \omega)$ to the point $\left(t_{x}^{(2)}, \omega\right)$ such that $\frac{d \phi_{x}}{d t}\left(t_{x}^{(2)}\right)=\omega_{x}+\alpha_{x} t_{x}^{(2)}=\omega$. This leads to:

$$
t_{x}^{(2)}=\frac{\omega-\omega_{x}}{\alpha_{x}}=\hat{t}_{x}(t, \omega)+\frac{\omega-\hat{\omega}_{x}(t, \omega)}{\alpha_{x}}+\frac{\nu_{x}}{\alpha_{x}} \operatorname{Im}\left(\tilde{t}_{x}(t, \omega)\right)
$$

which can be estimated by:

$$
\hat{t}_{x}^{(2)}(t, \omega)= \begin{cases}\frac{\omega-\hat{\omega}_{x}(t, \omega)+\operatorname{Im}\left(\hat{q}_{x}(t, \omega) \tilde{t}_{x}(t, \omega)\right)}{\operatorname{Im}\left(\hat{q}_{x}(t, \omega)\right)} & \text { if } \operatorname{Im}\left(\hat{q}_{x}(t, \omega)\right) \neq 0 \\ \hat{t}_{x}(t, \omega) & \text { otherwise }\end{cases}
$$

where $\hat{q}_{x}(t, \omega)$ is estimated using Eq. (29).

Finally, a new second-order horizontal synchrosqueezed Stransform is obtained using Eq. (19) by replacing the group delay estimator $\hat{t}_{x}(t, \omega)$ by $\hat{t}_{x}^{(2)}(t, \omega)$ given by Eq. (31).

\section{NUMERICAL EXPERIMENTS}

\section{A. Implemention issues}

Our implementation ${ }^{1}$ of the S-transform is based on Eq. (3) that is discretized as $\mathrm{ST}_{x}^{g}[k, m] \approx \mathrm{ST}_{x}^{g}\left(\frac{k}{F_{s}}, 2 \pi \frac{m F_{s}}{M}\right), F_{s}$ being the sampling frequency. The computation is based on the rectangle approximation method where $k \in \mathbb{Z}$ is the time sample index and $m \in \mathcal{M}$ is the discrete frequency bin. The number of frequency bins $M$ is chosen as an even number such as $\mathcal{M}=[-M / 2+1 ;-1] \cup[1 ; M / 2]$. Since a Gaussian function is defined on $]-\infty ;+\infty[$, a threshold $\Gamma$ is applied to obtain a finite number of time samples $K_{m}$ to be considered for the integration at frequency bin $m$ such as $\mathbf{e}^{-\frac{m^{2}\left(K_{m} / 2\right)^{2}}{2\left(f_{0} T\right)^{2} M^{2}}} \leq \Gamma$, which leads to:

$$
K_{m} \geq \frac{2 M f_{0} T \sqrt{2 \log (1 / \Gamma)}}{|m|} .
$$

Hence, a small value for $\Gamma$ leads to a large value for $K_{m}$ and an increase of the computation time. Our experiments use $\Gamma=10^{-4}$ which provides good results with a reasonable computation time in practice. When a signal reconstruction is required, a zero-padding of $2 K_{1}$ samples is applied to improve the reconstruction quality ( $c f$. Table I).

\section{B. TFR of a synthetic signal}

In this experiment, we analyze a synthetic multicomponent real-valued signal with 500 time samples, that is made of two impulses, one sinusoid, one chirp and one sinusoidally modulated sinusoid. Fig. 1 compares the resulting TFRs provided respectively by the STFT (Gabor transform) and the Stransform (i.e. rescaled Morlet wavelet transform) through the squared modulus of the original transform (i.e. spectrogram and stockwellogram) and the first- and second-order timereassigned horizontal synchrosqueezing transforms. Our computations use $M=600, f_{0} T=2$ and $L=T F_{s}=10$ for the STFT with a Signal-to-Noise Ratio (SNR) equal to $25 \mathrm{~dB}$ obtained by the addition of a Gaussian white noise. The TFRs provided by the existing methods are computed using the MATLAB implementions brought by the ASTRES toolbox [6]. The results show a clear improvement attested in terms of Rényi Entropy (RE) [9] (a lower RE corresponds to a better energy concentration of the TFR) provided by the second-order horizontal synchrosqueezing in comparison to the first-order version and the original transform TFRS for representing modulated components. Moreover, the new horizontal synchrosqueezed S-transform obtains a better RE in comparison to the STFT-based method on this multicomponent signal. This result can be explained by the two impulses which are almost perfectly located in the high frequencies due to the frequency-dependent resolution of the S-transform.

\footnotetext{
${ }^{1}$ Matlab codes freely available at https://fourer.fr/sthsst
} 


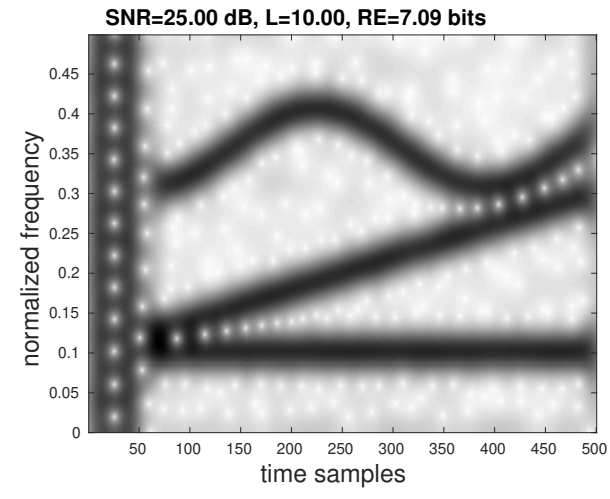

(a) spectrogram

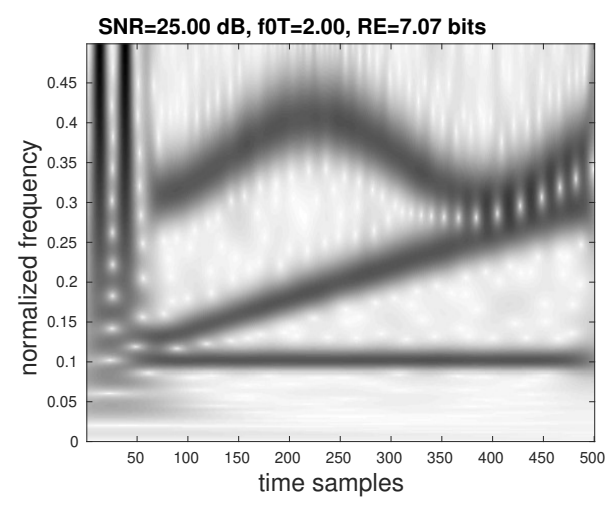

(d) stockwellogram

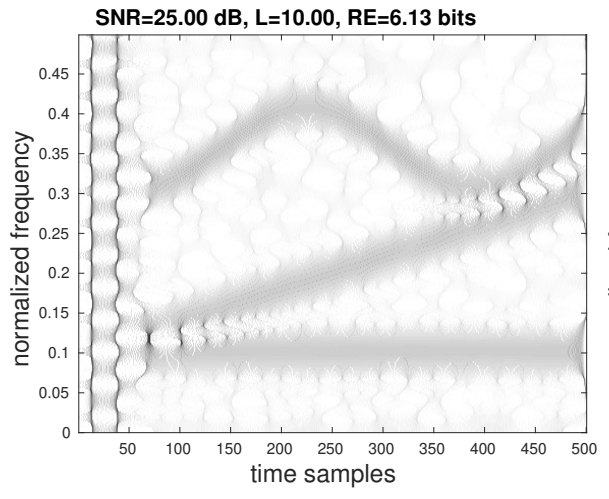

(b) time-reassigned synchrosqueezed STFT

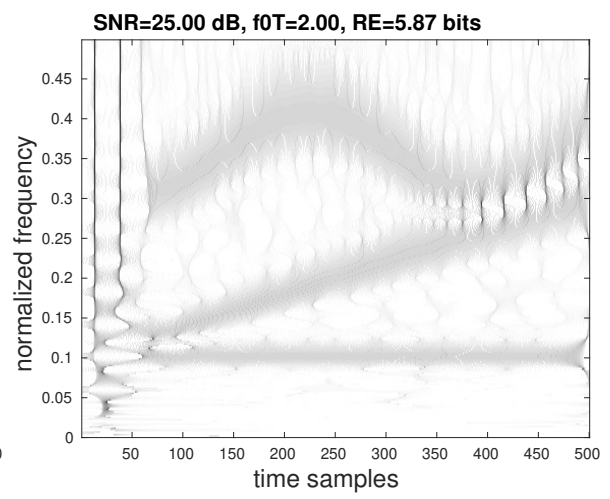

(e) time-reassigned synchrosqueezed S-transform

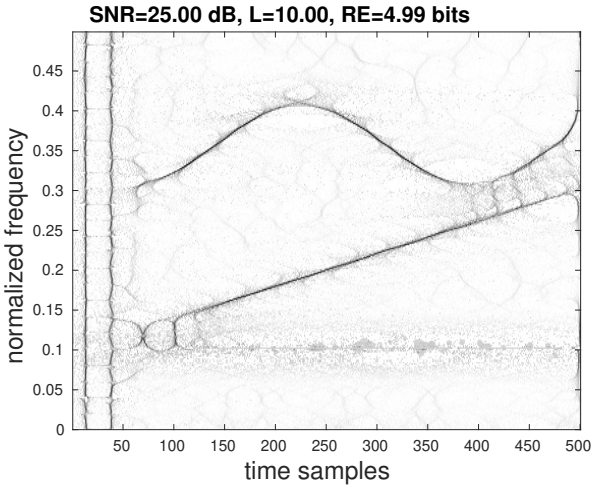

(c) second-order horizontal synchrosqueezed STFT

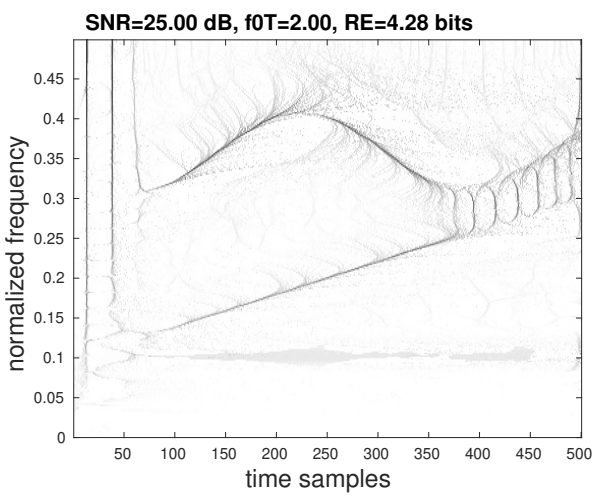

(f) second-order horizontal synchrosqueezed Stransform

Fig. 1. Comparison of the resulting TFRs with Rényi Entroy (at order $\alpha=3$ ) of a synthetic multicomponent signal. The TFRs of the synchrosqueezing methods (b),(c), (e) and (f) correpond to their squared modulus.

\section{Signal reconstruction results}

To assess the reconstruction capability of the proposed transforms, we compare in Table I the Reconstruction Quality Factor (RQF) defined by:

$$
\mathrm{RQF}(x, \hat{x})=10 \log _{10}\left(\frac{\sum_{k}|x[k]|^{2}}{\sum_{k}|x[k]-\hat{x}[k]|^{2}}\right),
$$

computed for the multicomponent signal presented in Fig. 1. As expected, the first- and second-order horizontal synchrosqueezed S-transforms obtain the same RQF with Eq. (22) as the S-transform using Eq. (9). The results show the effect of the number of frequency bins $M$ which should at least be equal to the length of the analyzed signal, and the effect of the threshold $\Gamma$ (b) which can increase the computation cost when too low and for which $10^{-2}$ is a suitable value.

Despite these reconstruction results are excellent (RQF above $50 \mathrm{~dB}$ ), they appear to be lower than those we previously obtained with the STFT-based horizontal synchrosqueezing (using a Gaussian window) when the same value of $M$ is used [8]. This could be explained by the logarithmic frequency resolution of the S-transform combined with the use of a uniform sampling of the frequency bins in our numerical implementation that is probably not optimal. This point may require further investigation that is scheduled in our future work.
TABLE I

RECONSTRUCTION QUALITY OF THE S-TRANSFORM, THE FIRST- AND THE SECOND-ORDER HORIZONTAL SYNCHROSQUEEZED S-TRANSFORMS USING THE SIGNAL PRESENTED IN FIG. 1 WITH $f_{0} T=2, M$ VARYING FROM 300 TO 2000 (A) AND $\Gamma$ VARYING FROM $10^{-1}$ TO $10^{-4}$ (B).

\begin{tabular}{|l|l|l|l|l|l|}
\hline \multirow{2}{*}{ (a) } & $M\left(\Gamma=10^{-4}\right)$ & 300 & 500 & 1000 & 2000 \\
& $\mathrm{RQF}(\mathrm{dB})$ & 0.85 & 54.10 & 60.12 & 66.14 \\
\hline \hline \multirow{2}{*}{ (b) } & $\Gamma(M=500)$ & $10^{-1}$ & $10^{-2}$ & $10^{-3}$ & $10^{-4}$ \\
& $\mathrm{RQF}(\mathrm{dB})$ & 53.45 & 54.10 & 54.10 & 54.10 \\
\hline
\end{tabular}

\section{CONCLUSION}

We have proposed a novel second-order time-reassigned synchrosqueezing of the S-transform based on an enhanced group delay estimator to deal with impulses and strongly modulated components. The proposed method can efficiently be computed using several S-transforms with specific analysis windows and presents interesting theoretical links with the STFT and the CWT. Our results show that the new proposed transform can obtain a better readability than the STFT-based horizontal synchrosqueezing for strongly modulated signals. In future work, we expect to further investigate the new proposed group delay estimator and to consider real-world application scenarios with a particular attention to propose numericallyefficient implementations. 


\section{REFERENCES}

[1] P. Flandrin, Time-Frequency/Time-Scale analysis. Acad. Press, 1998.

[2] K. Kodera, R. Gendrin, and C. de Villedary, "Analysis of time-varying signals with small BT values," IEEE Trans. Acoust., Speech, Signal Process., vol. 26, no. 1, pp. 64-76, Feb. 1978.

[3] F. Auger and P. Flandrin, "Improving the readability of time-frequency and time-scale representations by the reassignment method," IEEE Trans. Signal Process., vol. 43, no. 5, pp. 1068-1089, May 1995.

[4] I. Daubechies and S. Maes, "A nonlinear squeezing of the continuous wavelet transform," Wavelets in Medecine and Bio., pp. 527-546, 1996.

[5] F. Auger, P. Flandrin, Y. Lin, S. McLaughlin, S. Meignen, T. Oberlin, and $\mathrm{H}$. Wu, "TF reassignment and synchrosqueezing: An overview," IEEE Signal Process. Mag., vol. 30, no. 6, pp. 32-41, Nov. 2013.

[6] D. Fourer, J. Harmouche, J. Schmitt, T. Oberlin, S. Meignen, F. Auger, and P. Flandrin, "The ASTRES toolbox for mode extraction of nonstationary multicomponent signals," in Proc. EUSIPCO, Kos island, Greece, Aug. 2017, pp. 1170-1174.

[7] R. Souriau, D. Fourer, H.Chen, J. Lerbet, H. Maaref, and V. Vigneron, "High-voltage spindles detection from EEG signals using recursive synchrosqueezing transform," in Proc. GRETSI'19, Lille, France, Aug. 2019.

[8] D. Fourer and F. Auger, "Second-Order Time-Reassigned synchrosqueezing transform: Application to Draupner wave analysis," in Proc. EUSIPCO 2019, A Coruña, Spain, Sep. 2019.

[9] D.-H. Pham and S. Meignen, "High-order synchrosqueezing transform for multicomponent signals analysiswith an application to gravitationalwave signal," IEEE Transactions on Signal Processing, vol. 65, no. 12, pp. 3168-3178, 2017.

[10] T. Oberlin, S. Meignen, and V. Perrier, "Second-order synchrosqueezing transform or invertible reassignment? Towards ideal time-frequency representations," IEEE Trans. Signal Process., vol. 63, no. 5, pp. 13351344, Mar. 2015.
[11] D. Fourer, F. Auger, K. Czarnecki, Meignen, and Flandrin, "Chirp rate and instantaneous frequency estimation: Application to recursive vertical synchrosqueezing," IEEE Signal Process. Lett., vol. 24, no. 11, pp. 1724-1728, Nov. 2017.

[12] F. Auger, E. Chassande-Mottin, and P. Flandrin, "Making reassignment adjustable: the Levenberg-Marquardt approach," in Proc. IEEE ICASSP, Kyoto, Japan, Mar. 2012, pp. 3889-3892.

[13] D. Fourer, F. Auger, and P. Flandrin, "Recursive versions of the Levenberg-Marquardt reassigned spectrogram and of the synchrosqueezed STFT," in Proc. IEEE ICASSP, Shanghai, China, May 2016, pp. 4880-4884.

[14] R. Stockwell, L. Mansinha, and R. Lowe, "Localization of the complex spectrum: the S-transform," IEEE Trans. Signal Process., vol. 44, no. 4, pp. 998-1001, Apr. 1996

[15] D. He, H. Cao, S. Wang, and X. Chen, "Time-reassigned synchrosqueezing transform: The algorithm and its applications in mechanical signal processing," Mechanical Systems and Signal Processing, vol. 117, pp. 255-279, 2019

[16] J. C. Brown, "Calculation of a constant-Q spectral transform," The Journal of the Acoustical Society of America, vol. 89, no. 1, pp. 425434, 1991.

[17] D. Fourer, F. Auger, and J. Hu, "Reassigning and synchrosqueezing the Stockwell transform: Complementary proofs," Tech. Rep., Dec. 2015. [Online]. Available: http://hal.archives-ouvertes.fr/hal-01467244

[18] A. Grossmann and J. Morlet, "Decomposition of Hardy functions into square integrable wavelets of constant shape," IAM Journal of Mathematical Analysis, vol. 15, no. 4, pp. 723-736, 1984.

[19] S. Ventosa, C. Simon, M. Schimmel, J. Danobeitia, and A. Manuel, "The S-transform from a wavelet point of view," IEEE Trans. Signal Process., vol. 56, no. 7, pp. 2771-2780, Jul. 2008 